\title{
Comparison of Electrical Output Format Options for the Analysis of Strain-Gage Balance Calibration Data
}

\author{
N. Ulbrich ${ }^{\dagger}$ \\ Jacobs Technology Inc., Moffett Field, California 94035
}

\begin{abstract}
Fundamental characteristics of three gage output formats are discussed that may be used to both interpret and analyze wind tunnel strain-gage balance data. The first output format uses raw outputs, i.e., absolute voltage measurements, for the analysis. This choice requires an intercept term in the regression model of the outputs if the Iterative Method is chosen for the load prediction. Raw outputs can be used for the analysis of all known output characteristics as long as the Iterative Method is applied. However, raw outputs cannot be used to analyze data of a balance with bi-directional outputs if the Non-Iterative Method is chosen for the analysis. The second output format, i.e., difference type 1, uses the difference between raw outputs and the natural zeros of the balance gages for the analysis. In this case, the use of the intercept term becomes optional. Analysis results for difference type 1 will exactly match analysis results obtained by using raw outputs as long as identical math models are used for the regression analysis. In addition, difference type 1 may be used to analyze data of a balance with bi-directional outputs if an analyst prefers to process data using the NonIterative Method. The third output format, i.e., difference type 2, uses the difference between raw outputs and the outputs of the zero load point of a load series for the data analysis. The application of this format is only recommended if the maximum magnitude of all tare loads of a given balance calibration data set is less than two percent of capacity. Data from the calibration of a force balance is used to illustrate the application of the three gage output formats.
\end{abstract}

\section{Nomenclature}

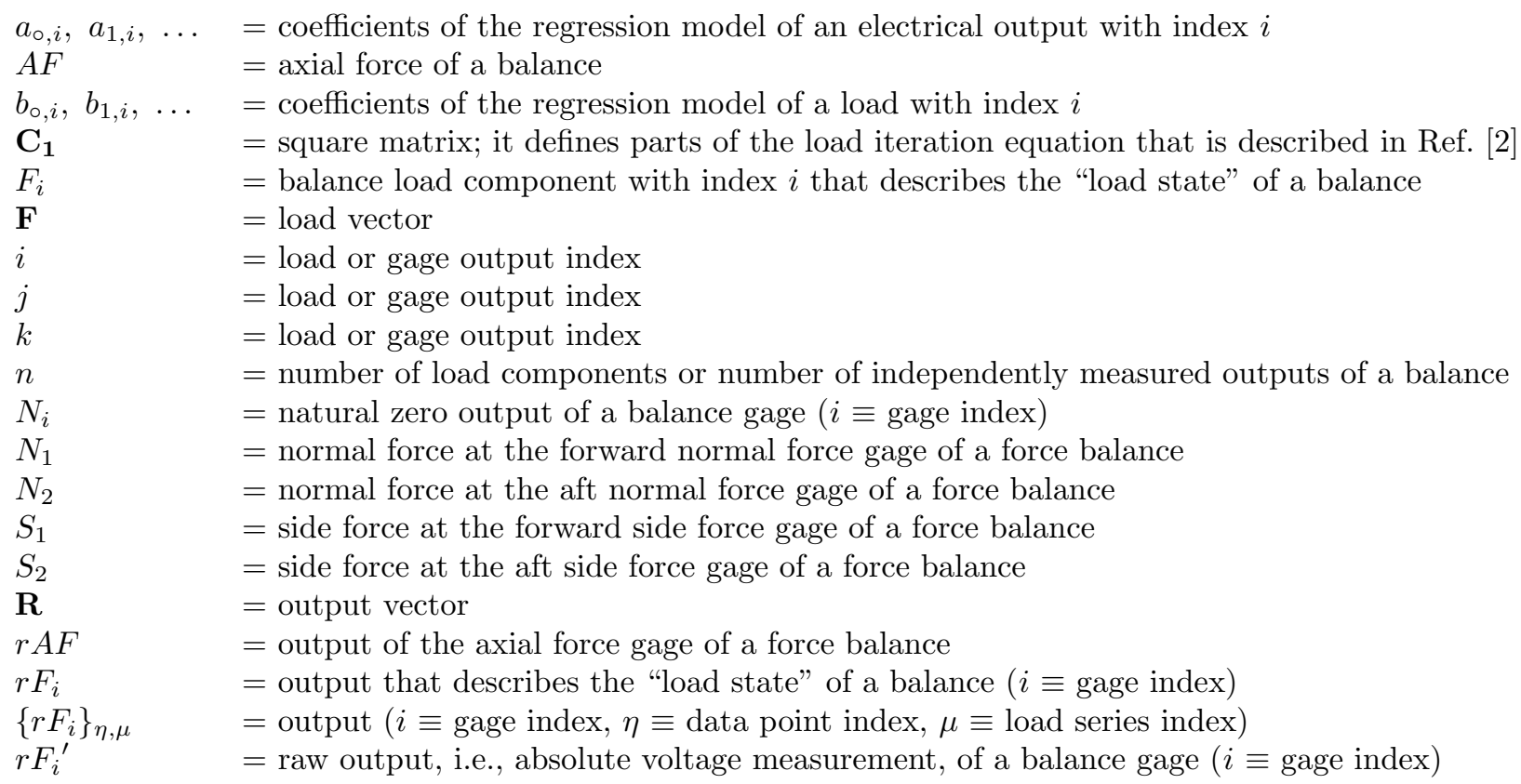

\footnotetext{
$\nmid$ Aerodynamicist, Jacobs Technology Inc.
} 


$\begin{array}{ll}\left\{r F_{i}^{\prime}\right\}_{\eta, \mu} & =\text { raw output }(i \equiv \text { gage index, } \eta \equiv \text { data point index, } \mu \equiv \text { load series index }) \\ R M & =\text { rolling moment of a balance } \\ r N 1 & =\text { output of the forward normal force gage of a force balance } \\ r N 2 & =\text { output of the aft normal force gage of a force balance } \\ r R M & =\text { output of the rolling moment gage of a force balance } \\ r S 1 & =\text { output of the forward side force gage of a force balance } \\ r S 2 & =\text { output of the aft side force gage of a force balance } \\ \left\{Z_{i}\right\}_{\mu} & =\text { raw output of a zero load point of a load series }(i \equiv \text { gage index, } \mu \equiv \text { load series index }) \\ \eta & =\text { data point index } \\ \mu & =\text { load series index } \\ \xi_{i} & =\text { relationship between a gage output and the load set of a balance }(i \equiv \operatorname{gage~index~}) \\ \psi_{i} & =\text { relationship between a load and the gage output set of a balance }(i \equiv \text { load index })\end{array}$

\section{Introduction}

In general, a strain-gage balance is used during a wind tunnel test to predict the forces and moments that act on the wind tunnel model from the measured strain-gage outputs of the balance. The required mathematical relationship between loads and outputs is obtained by analyzing balance calibration data that is recorded by applying a set of known loads and measuring the resulting outputs of the balance gages.

Both the format of the balance loads and the format of the electrical outputs have an influence on the overall characteristics of the balance load prediction method that an analyst chooses. For example, loads of a force balance could be expressed in either force balance or direct-read format before an analysis takes place. This selection influences the convergence characteristics of the chosen load iteration equation assuming that the Iterative Method is used for the balance load prediction (see, e.g., Refs. [1] to [4] for a detailed description of the Iterative Method). The overall load prediction accuracy is improved if tare corrected instead of applied loads are used for the regression analysis of the given balance calibration data (see Ref. [5] for a detailed discussion of balance tare load corrections). The format of the electrical outputs also influences characteristics and accuracy of the chosen load prediction method. Unfortunately, output format options are rarely discussed in the literature as analysts often only use a single output format for all data analysis tasks. Therefore, the author decided to discuss advantages and disadvantages of three possible gage output formats in an attempt to close this gap in the existing literature.

The discussion of the influence of an analyst's output format choice on the balance load prediction requires some understanding of the different load prediction methods that are used in the aerospace testing community. Therefore, basic elements and assumptions supporting the Iterative and Non-Iterative Method are discussed in the next section of the paper. Then, three frequently used electrical output format options are presented. Finally, data from a typical six-component wind tunnel balance is used to illustrate the application of the three output formats.

\section{Balance Load Prediction Methods}

\section{A. General Remarks}

Different methods are used in the aerospace testing community to predict balance loads from measured strain-gage outputs during a wind tunnel test. They may be separated into two classes: Iterative and NonIterative Methods (see Refs. [1] to [4] for more detail). Both classes fit a given set of balance calibration data in the least squares sense and use the result of the analysis for the prediction of balance loads from the measured outputs. The load prediction methods differ in the selection of the independent and dependent variables that are used for the regression analysis of the balance calibration data. The Iterative Method, for example, treats balance loads as independent variables and gage outputs as dependent variables during the regression analysis. In other words, gage outputs are fitted as a function of the balance loads. Therefore, this method needs to construct a load iteration scheme from the result of the regression analysis so that loads can be predicted from measured gage outputs during a wind tunnel test. The Non-Iterative Method, on the other hand, treats balance loads as dependent variables and gage outputs as independent variables during the regression analysis. It directly fits loads as a function of measured gage outputs. Consequently, no iteration is needed to predict loads from gage outputs during a wind tunnel test. Table 1 below summarizes 
variable choices for the two load prediction methods.

Table 1: Variable choices for strain-gage balance load prediction methods.

\begin{tabular}{|c|c|c|}
\hline & Iterative Method & Non-Iterative Method \\
\hline \hline Independent Variables & loads (forces \& moments) & strain-gage outputs \\
\hline Dependent Variables & strain-gage outputs & loads (forces \& moments) \\
\hline
\end{tabular}

It is helpful for a better understanding of the two load prediction methods to describe the "load state" of the balance by using a control volume (see Ref. [6]). The input variables of the control volume are the "loads" that act on the metric part of the balance. They can be described in vector format as follows ...

$$
\text { Load Vector } \Longrightarrow \mathbf{F}=\left[\begin{array}{c}
F_{1} \\
\vdots \\
F_{i} \\
\vdots \\
F_{n}
\end{array}\right] \quad ; \quad 1 \leq i \leq n
$$

where $F_{i}$ is a load component and $n$ is the total number of load components that independently act on the metric part. The output variables of the control volume are the measured electrical outputs (or output combinations) of the gages that "exit" the control volume through the wire harness. Again, the output variables can be described in vector format as follows ...

$$
\text { Output Vector } \Longrightarrow \mathbf{R}=\left[\begin{array}{c}
r F_{1} \\
\vdots \\
r F_{i} \\
\vdots \\
r F_{n}
\end{array}\right] \quad ; \quad 1 \leq i \leq n
$$

where $r F_{i}$ is a strain-gage output and $n$ is the total number of gage outputs that independently respond to loads acting on the metric part.

Now, let us assume that (i) the load vectors define a "load space" and (ii) the output vectors define an "output space." Each load vector identifies a point in the $n$-dimensional "load space" that describes a "load state" of the balance. Similarly, each output vector identifies a point in the $n$-dimensional "output space" that describes a "load state" of the balance. Consequently, the balance load prediction will only work reliably if a load vector describing a specific "load state" of the balance in the "load space" is "uniquely" mapped to an output vector that describes the same "load state" in the "output space" (and vice versa). In other words, a robust load prediction method establishes a "unique" mapping between the load and output spaces such that two different descriptions of one specific "load state" of the balance get connected.

Figure 1a below shows the situation from the viewpoint of the Iterative Method. In this case, the regression analysis first defines a mapping from the load space to the output space. Then, an iteration process is defined from the regression analysis results. The iteration process "reverses" the mapping so that

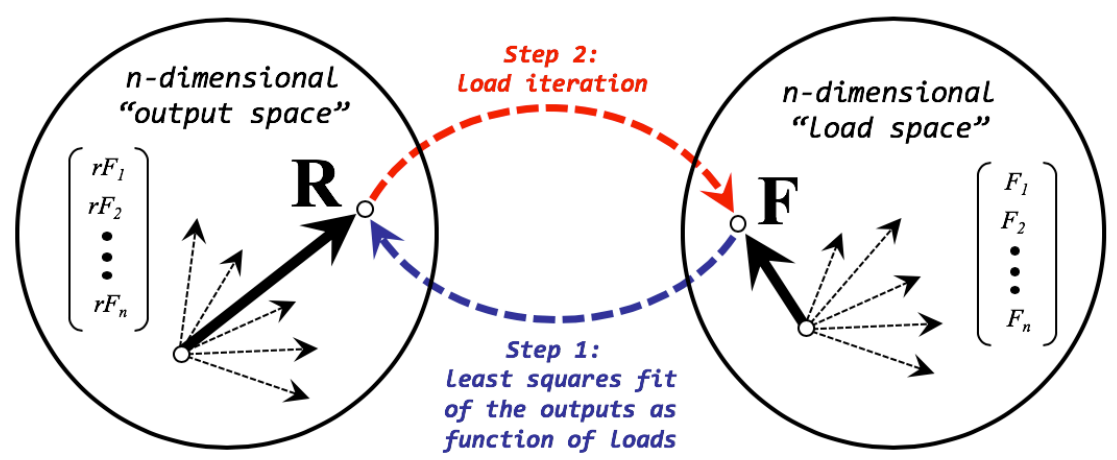

Fig. 1a Two step balance load prediction process of the Iterative Method. 
loads can be predicted from outputs during a wind tunnel test. Figure $1 \mathrm{~b}$ shows the situation from the viewpoint of the Non-Iterative Method. Now, the situation is much simpler. The regression analysis defines a mapping from the output space to the load space that can directly be used to predict loads from outputs during a wind tunnel test.

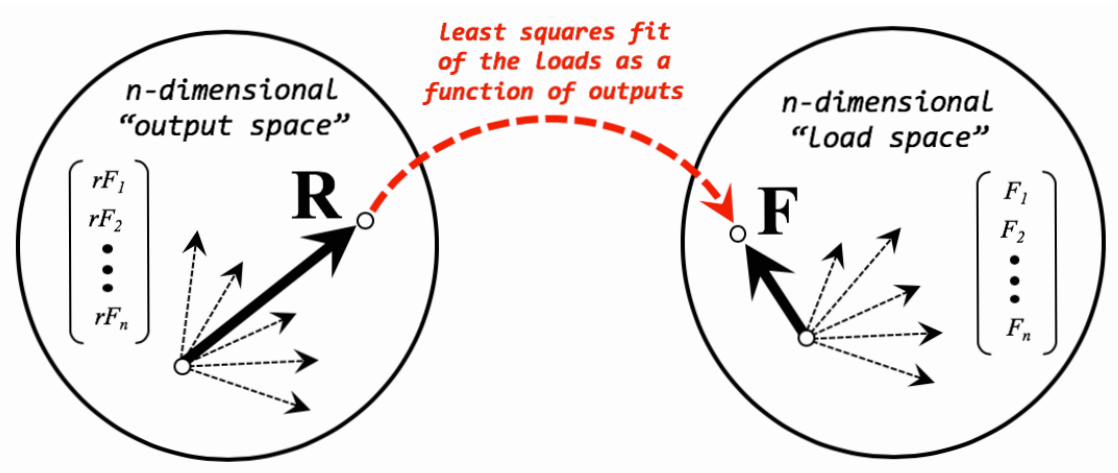

Fig. 1b Single step balance load prediction process of the Non-Iterative Method.

Both the components of the load vector and the components of the output vector have uncertainties associated with them. In particular, the uncertainty of the load vector will depend on the mathematical mapping between the load and output space whenever a tare load iteration is applied. However, the total objective "information" contained in a given calibration data set about the physical behavior of the balance is independent of the chosen load prediction method if small differences in the predicted tare loads are ignored. This fundamental conclusion is confirmed by the author's past experience. He consistently observed that the load prediction accuracy of the Iterative Method is as good as the load prediction accuracy of the NonIterative Method as long as (i) similar regression models of the calibration data are used to construct the mapping from load space to output space (or vice versa) and (ii) the terms of the regression models do not have hidden linear or massive near-linear dependencies. Characteristics of the different load prediction methods are reviewed in the next sections of the paper in order to better understand the influence of the output format on the load predictions.

\section{B. Iterative Method}

The Iterative Method uses a two-step approach to determine loads from gage outputs assuming that the physical characteristics of the balance are defined by the given calibration data. First, outputs are fitted as a function of loads (Step 1). Then, loads are predicted using a load iteration equation that results from the fit of the outputs (Step 2). In theory, Step 1, i.e., the mapping from "load space" to "output space," can be described for each gage output by using the following function:

$$
r F_{i}=\xi_{i}\left(F_{1}, F_{2}, \ldots, F_{n}\right)
$$

The exact form of the function $\xi_{i}$ is unknown as a real-world balance has very complex load paths from the metric to the non-metric part of the balance. However, it is possible to approximate the unknown functional relationship $\xi_{i}$ between the electrical output $r F_{i}$ and the load component set $F_{1}, \ldots, F_{n}$ by using a multi-variate Taylor Series that is developed in the neighborhood of zero absolute load. Then, we get the following approximation of the unknown mapping function $\xi_{i}$

$$
r F_{i}=\xi_{i}\left(F_{1}, \ldots, F_{n}\right) \approx \underbrace{a_{\circ, i}+a_{1, i} \cdot F_{1}+a_{2, i} \cdot F_{2}+a_{3, i} \cdot F_{3}+\cdots}_{\text {Taylor Series centered at zero absolute load }}
$$

The coefficients $a_{\circ, i}, a_{1, i}, \ldots$ of the Taylor Series approximation are obtained by fitting the output $r F i$ of the gage with index $i$ as a function of the given balance loads. By design, the fit is a multi-variate linear fit that can easily be solved by applying a linear system solver to the normal equations of the least squares 
problem. The right-hand side of Eq. (3b) uses different types of regression model terms depending on the chosen Taylor Series approximation. For example, the following three math term groups are used if a second order Taylor Series is selected (excluding the intercept):

$$
\text { Taylor Series (second order) } \Longrightarrow F_{i},\left\{F_{i}\right\}^{2}, F_{i} \cdot F_{j}
$$

Similarly, six math term groups are used if a third order Taylor Series is chosen for the analysis:

$$
\text { Taylor Series (third order) } \Longrightarrow F_{i},\left\{F_{i}\right\}^{2},\left\{F_{i}\right\}^{3}, F_{i} \cdot F_{j}, F_{i} \cdot\left\{F_{j}\right\}^{2}, F_{i} \cdot F_{j} \cdot F_{k}
$$

It is also possible to combine terms of a second or third order Taylor Series with other types of terms that model design specific output characteristics of balance gages. This approach was chosen by AIAA's Internal Balance Working Group (see Ref. [2]). In that case, terms defined by a multi-variate Taylor Series are used side-by-side with absolute value terms of the loads so that any bi-directional behavior of a balance output can mathematically be described. Bi-directional behavior is typically observed for the normal and side force gage outputs of ABLE/TASK balances. Consequently, the following "hybrid" regression model of the outputs consisting of twelve math term groups may be used for the analysis:

$$
\text { Hybrid } \Longrightarrow\left\{\begin{array}{c}
F_{i},\left\{F_{i}\right\}^{2},\left\{F_{i}\right\}^{3}, F_{i} \cdot F_{j}, F_{i} \cdot\left\{F_{j}\right\}^{2}, F_{i} \cdot F_{j} \cdot F_{k} \equiv \text { Taylor Series terms } \\
\left|F_{i}\right|, F_{j} \cdot\left|F_{j}\right|,\left|F_{i} \cdot F_{j}\right|, F_{i} \cdot\left|F_{j}\right|,\left|F_{i}\right| \cdot F_{j},\left|\left\{F_{i}\right\}^{3}\right| \equiv \text { absolute value terms }
\end{array}\right.
$$

It must be pointed out that the third order Taylor Series term combination chosen by AIAA's Internal Balance Working Group is "incomplete" because the cross-product terms $F_{i} \cdot\left\{F_{j}\right\}^{2}$ and $F_{i} \cdot F_{j} \cdot F_{k}$ were omitted. Therefore, AIAA's regression model of the gage outputs uses only ten of the twelve math term groups that are listed above (AIAA's ten math term groups are defined in Ref. [2], Eq. (3.1.3)).

It is important to mention that the above list of twelve math term groups simply defines all terms that could potentially be used for the regression model of an output. It is ultimately the analyst's responsibility (i) to determine which subset of terms is truly supported by the balance calibration data and (ii) to avoid linear or massive near-linear dependencies in the regression models of the outputs. The term selection should be made by an analyst's "subject matter knowledge" in combination with the use of metrics like the "variance inflation factor" of a regression model term (see, e.g., Ref. [5] for details about the "variance inflation factor").

It remains to construct a load iteration equation from the regression model of the outputs that is defined in Eq. (3b) so that loads can be predicted from measured outputs during a wind tunnel test (see also Step 2 in Fig. 1a). The derivation of the load iteration equation is complex. In addition, a detailed understanding of the load iteration equation is not needed in order to evaluate advantages and disadvantages of different gage output format options that may be used for the processing of balance data. Therefore, the discussion of the load iteration equation is omitted in the paper because it is already described in great detail in the literature (see, e.g., Refs. [2], [3], and [4]).

\section{Non-Iterative Method}

Now, characteristics of the Non-Iterative Method are reviewed. This method uses a one-step approach to determine loads from gage outputs. In other words, existing balance calibration data is analyzed by directly fitting loads as a function of outputs. Theoretically, this mapping from "output space" to "load space" can be described for each load component by using the following function:

$$
F_{i}=\psi_{i}\left(r F_{1}, r F_{2}, \ldots, r F_{n}\right)
$$

Again, as it was the case for the Iterative Method, the exact form of the mapping function $\psi_{i}$ is unknown as real-world balances have very complex load paths from the metric to the non-metric part. However, it is possible to approximate the unknown function $\psi_{i}$ between the load component $F_{i}$ and the electrical output set $r F_{1}, \ldots, r F_{n}$ by using a multi-variate Taylor Series that is developed in the neighborhood of the outputs for zero absolute load. Then, we get the following approximation for the unknown mapping function $\psi_{i}$

$$
F_{i}=\psi_{i}\left(r F_{1}, \ldots, r F_{n}\right) \approx \underbrace{b_{\circ, i}+b_{1, i} \cdot r F_{1}+b_{2, i} \cdot r F_{2}+b_{3, i} \cdot r F_{3}+\cdots}_{\text {Taylor Series centered at outputs of zero absolute load }}
$$


The coefficients $b_{\circ, i}, b_{1, i}, \ldots$ of the Taylor Series approximation are obtained by fitting the load $F_{i}$ with index $i$ as a function of the measured gage output set. By design, the fit is a multi-variate linear fit that can easily be solved by applying a linear system solver to the normal equations of the least squares problem. The right-hand side of Eq. (4b) uses different types of regression model terms depending on the chosen Taylor Series approximation. For example, the following three math term groups are used if the Taylor Series is truncated after the second order terms (excluding the intercept):

$$
\text { Taylor Series (second order) } \Longrightarrow r F_{i},\left\{r F_{i}\right\}^{2}, r F_{i} \cdot r F_{j}
$$

Similarly, six math term groups are used for analysis if the series is truncated after the third order terms:

$$
\text { Taylor Series (third order) } \Longrightarrow r F_{i},\left\{r F_{i}\right\}^{2},\left\{r F_{i}\right\}^{3}, r F_{i} \cdot r F_{j}, r F_{i} \cdot\left\{r F_{j}\right\}^{2}, r F_{i} \cdot r F_{j} \cdot r F_{k}
$$

It is also possible to combine terms of a second or third order Taylor Series with terms that model design specific characteristics of a balance. Then, after extending the approach chosen by AIAA's Internal Balance Working Group to the Non-Iterative Method, terms defined by a Taylor Series are used side-by-side with absolute value terms of the outputs to potentially describe the bi-directional behavior of a balance. Consequently, the following hybrid math term group combination may be used for the analysis:

$$
\text { Hybrid } \Longrightarrow\left\{\begin{array}{c}
r F_{i},\left\{r F_{i}\right\}^{2},\left\{r F_{i}\right\}^{3}, r F_{i} \cdot r F_{j}, r F_{i} \cdot\left\{r F_{j}\right\}^{2}, r F_{i} \cdot r F_{j} \cdot r F_{k} \equiv \text { Taylor Series terms } \\
\left|r F_{i}\right|, r F_{j} \cdot\left|r F_{j}\right|,\left|r F_{i} \cdot r F_{j}\right|, r F_{i} \cdot\left|r F_{j}\right|,\left|r F_{i}\right| \cdot r F_{j},\left|\left\{r F_{i}\right\}^{3}\right| \equiv \text { absolute value terms }
\end{array}\right.
$$

The Non-Iterative Method has the advantage that it is a one-step method. No iteration is needed to compute loads from measured strain-gage outputs during a wind tunnel test. Therefore, the balance loads can directly be computed by using the right-hand side of Eq. (4b) above as soon as the regression coefficients $b_{\circ, i}, b_{1, i}, \ldots$ are known for each balance load component.

It is important to realize that the above list of twelve math term groups simply defines all terms that could potentially be used for the regression model of a load. It is ultimately the analyst's responsibility (i) to determine which subset of terms is truly supported by the balance calibration data and (ii) to avoid linear or massive near-linear dependencies in the regression models of the loads. The term selection should be made by an analyst's "subject matter knowledge" in combination with the use of metrics like the "variance inflation factor" of a regression model term (see, e.g., Ref. [5] for details about the "variance inflation factor").

\section{Electrical Output Format Options}

Three different gage output format options exist that may be used to analyze strain-gage balance data. The first output format option uses "raw outputs", i.e., absolute voltage measurements, directly as input for the analysis of strain-gage balance data. Consequently, the format is defined as follows ...

$$
\text { Raw Output } \Longrightarrow\left\{r F_{i}\right\}_{\eta, \mu}=\left\{r F_{i}{ }^{\prime}\right\}_{\eta, \mu}
$$

where $\left\{r F_{i}{ }^{\prime}\right\}_{\eta, \mu}$ is the raw output of the balance gage ( $i \equiv$ gage index, $\eta \equiv$ data point index, $\mu \equiv$ load series index). The use of raw outputs makes the preparation of the data input file "trivial" as outputs are directly used as supplied. In addition, the natural zeros are the raw outputs of the balance in an assumed "weightless" condition. These non-zero values are the electrical representation of zero absolute load. They are needed for the tare load iteration (see Ref. [5] for a description of the tare load iteration process). Consequently, assuming that the Iterative Method is used for the balance calibration data analysis, the intercept term must always be included in the regression model of the outputs as it is a least squares approximation of the natural zeros. Raw outputs can be used to analyze balance calibration data of all balance types with all known output characteristics as long as an analyst applies the Iterative Method. However, raw outputs cannot be used to analyze calibration data of balances with bi-directional outputs if the Non-Iterative Method is chosen for the analysis. This restriction results from the fact that the traditionally used math term groups in the regression model of a load component do not allow for a constant output shift (this limitation is discussed in the appendix; see also Ref. [7] for a discussion of balances with bi-directional output characteristics). In conclusion, characteristics and application of "raw outputs" can be summarized as follows: 
- defined as an absolute voltage measurement that uses zero volts as a datum;

- data preparation is "trivial" as raw outputs are directly used as supplied;

- "raw output" can be used to analyze data of balances with all known gage output characteristics as long as the Iterative Method is applied;

- "raw output" cannot be used to analyze data of balances with bi-directional gage output characteristics whenever the Non-Iterative Method is applied;

- natural zeros are the "raw outputs" of a balance in a "weightless" condition;

- natural zeros could also be obtained from "raw outputs" of related load series;

- Iterative Method $\Longrightarrow$ intercepts are a least squares description of the natural zeros;

- Iterative Method $\Longrightarrow$ intercepts must be used in regression models of "raw outputs";

- tare loads can be obtained from output data by using a tare load iteration;

- "raw output" can be used to analyze data of both primary \& auxiliary balances;

The second output format option, i.e., Difference Type 1, uses the difference between the raw output and the natural zero of a balance gage for the analysis. Consequently, the format is defined as follows ...

Difference Type $1 \Longrightarrow$ transformed raw outputs $\Longrightarrow\left\{r F_{i}\right\}_{\eta, \mu}=\left\{r F_{i}\right\}_{\eta, \mu}-N_{i}$

where $\left\{r F_{i}{ }^{\prime}\right\}_{\eta, \mu}$ is the raw output and $N_{i}$ is the natural zero of the balance gage $(i \equiv$ gage index, $\eta \equiv$ data point index, $\mu \equiv$ load series index). The use of Difference Type 1 requires a subtraction of the natural zeros from all raw outputs. In other words, it is a simple linear transformation (output shift) that is applied to the raw outputs. Consequently, the transformed values of all natural zeros are "zeros". These "zeros" are the updated electrical representation of zero absolute load (they are needed as data inputs if a tare load iteration is performed using the transformed outputs). Therefore, assuming that the Iterative Method is used for the balance calibration data analysis, the intercept term is "optional" in the regression model of the outputs as it is a least squares approximation of zero, i.e., of the "transformed" original natural zeros. Regression analysis results for Difference Type 1 will exactly match corresponding analysis results obtained for the raw outputs if (i) an intercept term is included in the math models and (ii) the same math model is used for the analysis. Difference Type 1 can be used to analyze balance calibration data of all balance types with all known output characteristics using either the Iterative or the Non-Iterative Method. In particular, Difference Type 1 can be used in combination with the Non-Iterative Method to analyze data of balances with bi-directional outputs. Finally, characteristics and use of Difference Type 1 can be summarized as follows:

Difference Type $1 \Longrightarrow$ Characteristics and Application

- defined as difference between raw outputs and natural zeros of the balance;

- data preparation requires subtraction of natural zeros from all outputs;

- Difference Type 1 can be used to analyze data of balances with all known gage output characteristics as long as the Iterative Method is applied;

- Difference Type 1 must be used to analyze data of balances with bi-directional gage output characteristics whenever the Non-Iterative Method is applied;

- natural zeros equal "zeros" in "transformed" output space of the balance;

- Iterative Method $\Longrightarrow$ intercepts are a least squares description of "zero" output;

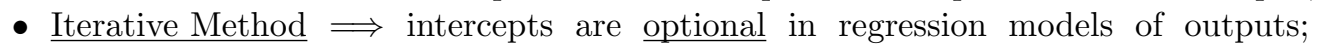

- tare loads can be obtained from output data by using a tare load iteration;

- Difference Type 1 can be used to analyze primary \& auxiliary balance data;

- analysis results match those for "raw output" if the same math terms are used;

The third output format option, i.e., Difference Type 2, uses the difference between the raw output and the output of the zero load point of the load series of the data point for the analysis. The zero load point 
is the data point whose electrical outputs are exclusively caused by (i) the weight of the metric part of the balance, (ii) the weight of the calibration body, and (iii) the weight of all calibration hardware pieces (flexures, yokes, threaded rods, weight pans, etc.). Consequently, the format is defined as follows ...

$$
\text { Difference Type } 2 \Longrightarrow \text { transformed raw outputs } \Longrightarrow\left\{r F_{i}\right\}_{\eta, \mu}=\left\{r F_{i}^{\prime}\right\}_{\eta, \mu}-\left\{Z_{i}\right\}_{\mu}
$$

where $\left\{r F_{i}{ }^{\prime}\right\}_{\eta, \mu}$ is the raw output of the balance gage and $\left\{Z_{i}\right\}_{\mu}$ is the output of the zero load point of the load series that a data point belongs to $(i \equiv$ gage index, $\eta \equiv$ data point index, $\mu \equiv$ load series index). Difference Type 2 requires a subtraction of the outputs of the zero load point of a load series from the raw outputs of the given data set. Therefore, the data input file preparation for the use of Difference Type 2 is the most complex of the three format choices. Difference Type 2, like Difference Type 1, is a simple output transformation. However, Difference Type 2 subtracts the electrical outputs of a "local" load datum, i.e., the output of the zero load point of the load series, from the raw outputs. Difference Type 1, on the other hand, subtracts the electrical outputs of a "global" load datum, i.e., the natural zeros, from the raw outputs. The transformed values of all natural zeros are "zeros". These "zeros" are the updated electrical representation of zero absolute load. Again, assuming that the Iterative Method is used, the intercept term is "optional" in the regression model of the outputs.

Difference Type 2 can be used to analyze balance calibration data of all balance types with all known output characteristics as long as the maximum magnitude of the tare loads of all load series of the given data is less than the empirical threshold of $\approx 2 \%$ of load capacity. It is important to point out that, by design, Difference Type 2 assumes small/negligible tare loads. Therefore, analysis results for Difference Type 2 are not as accurate as results that can be obtained by using either Difference Type 1 or Raw Output for the data analysis as those two alternate gage output formats support a tare load iteration. Now, characteristics and use of Difference Type 2 can be summarized as follows:

\section{Difference Type $2 \Longrightarrow$ Characteristics and Application}

- defined as difference between raw outputs and zeros load outputs of load series

- data preparation is "complex" as output subtraction must be done series by series

- by design, tare loads are zero as transformed outputs imply negligible tare loads

- Difference Type 2 may be used if the magnitude of all tare loads is below $2 \%$ of capacity

- "transformed" natural zeros equal "zeros" in "transformed" output space

- Iterative Method $\Longrightarrow$ intercepts are a least squares description of "zero" output

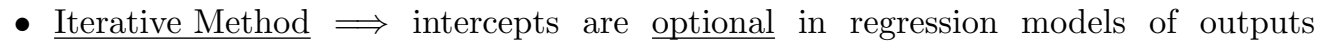

It is interesting to compare different processing options for balance data. Table 2 below lists all five options that are possible by using the three output formats that are defined in this section.

Table 2: Processing options for strain-gage balance data.

\begin{tabular}{|c|c|c|c|c|c|}
\hline Option & Raw Output & Difference Type 1 & Difference Type 2 & Intercept & Comments \\
\hline 1 & $x$ & - & - & $x$ & $\begin{aligned} \text { natural zeros } & \neq 0 \\
\text { tare loads } & \neq 0\end{aligned}$ \\
\hline 2 & - & $x$ & - & $x$ & $\begin{aligned} \text { natural zeros } & =0 \\
\text { tare loads } & \neq 0\end{aligned}$ \\
\hline 3 & - & $x$ & - & - & $\begin{array}{r}\text { natural zeros }=0 \\
\text { tare loads } \neq 0\end{array}$ \\
\hline 4 & - & - & $x$ & $x$ & $\begin{aligned} \text { natural zeros } & =0 \\
\text { tare loads } & =0\end{aligned}$ \\
\hline 5 & - & - & $x$ & - & $\begin{aligned} \text { natural zeros } & =0 \\
\text { tare loads } & =0\end{aligned}$ \\
\hline
\end{tabular}

American Institute of Aeronautics and Astronautics 
Calibration data of NASA's MK40A force balance is discussed in the next section of the paper to illustrate typical results for some of the analysis options that are listed in Table 2 above.

\section{Discussion of Example}

\section{A. General Remarks}

Data from a manual calibration of the NASA's MK40A balance was selected to illustrate the application of the three different gage output formats to real-world data. The MK40A is a six-component force balance of ABLE/TASK design that measures five forces and one moment $(N 1, N 2, S 1, S 2, A F, R M)$. It has a diameter of 2.5 inches $(63.5 \mathrm{~mm})$ and a total length of 17.31 inches $(439.7 \mathrm{~mm})$. Table 3 below lists the load capacity of each load component in English engineering units.

Table 3: Load capacities of the NASA Ames MK40A force balance.

\begin{tabular}{|c|c|c|c|c|c|}
\hline$N 1, l b f$ & $N 2, l b f$ & $S 1, l b f$ & $S 2, l b f$ & $R M$, in-lbf & $A F, l b f$ \\
\hline \hline 3500 & 3500 & 2500 & 2500 & 400 & 8000 \\
\hline
\end{tabular}

The calibration of the balance was performed by using gravity weights. A total of 164 data points were recorded that were distributed across 16 load series. Calibration data input files were prepared to support the five processing options listed in Table 2 by using the applied loads and the three possible gage output formats, i.e., Raw Output, Difference Type 1, and Difference Type 2, as input.

Figure 2 below shows the natural zeros of the balance gages and parts of the original calibration data input file of the balance when the outputs were described in the format Raw Output.

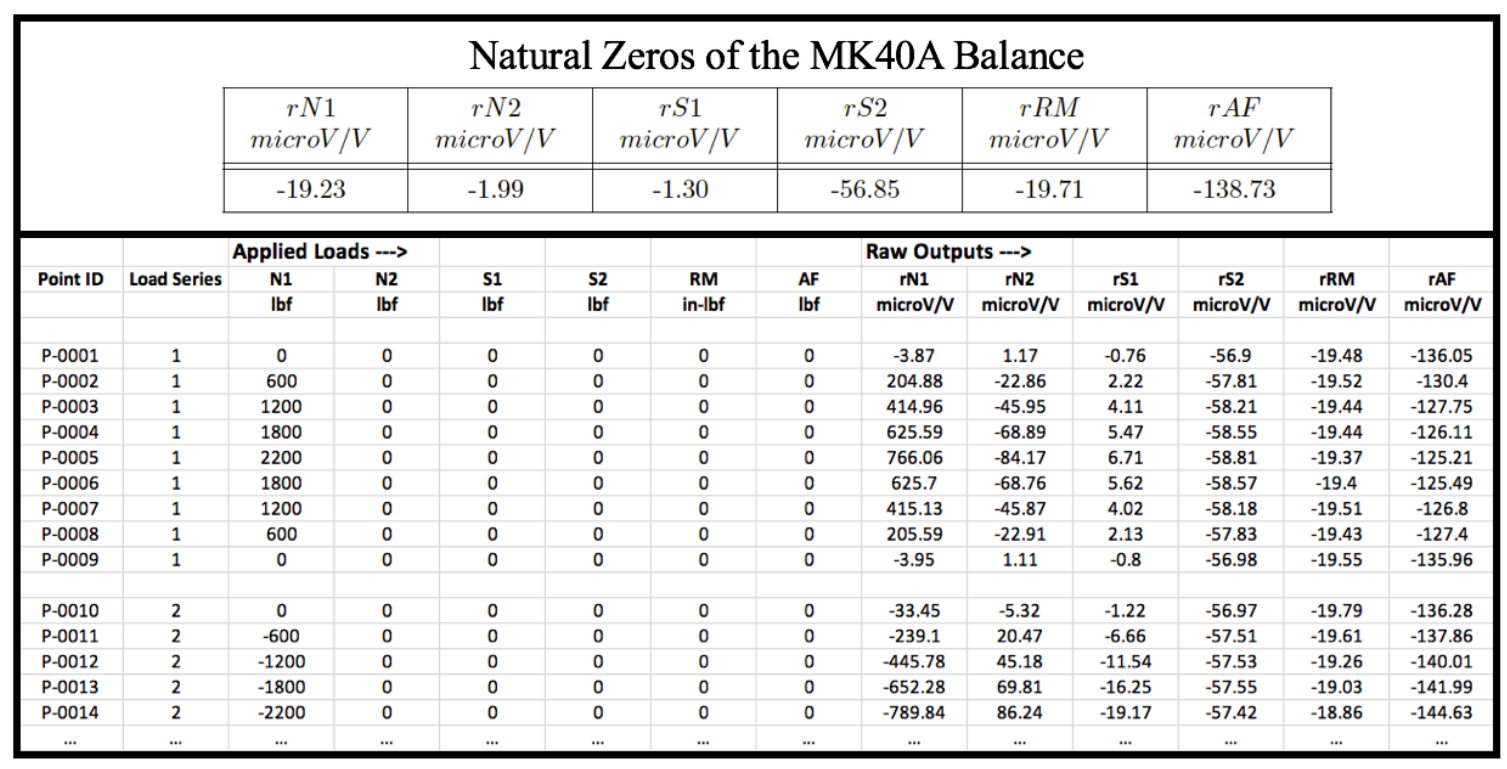

Fig. 2 Natural zeros and original calibration data of the MK40A balance if Raw Outputs describe the "load state" of the balance in the "output space."

Figure 3 below shows the transformed natural zeros of the balance gages and parts of the revised calibration data input file of the balance when outputs were described in the format Difference Type 1. The last six data columns of Fig. 3 were obtained by subtracting the natural zeros given in the header of Fig. 2 from the last six data columns of Fig. 2. 


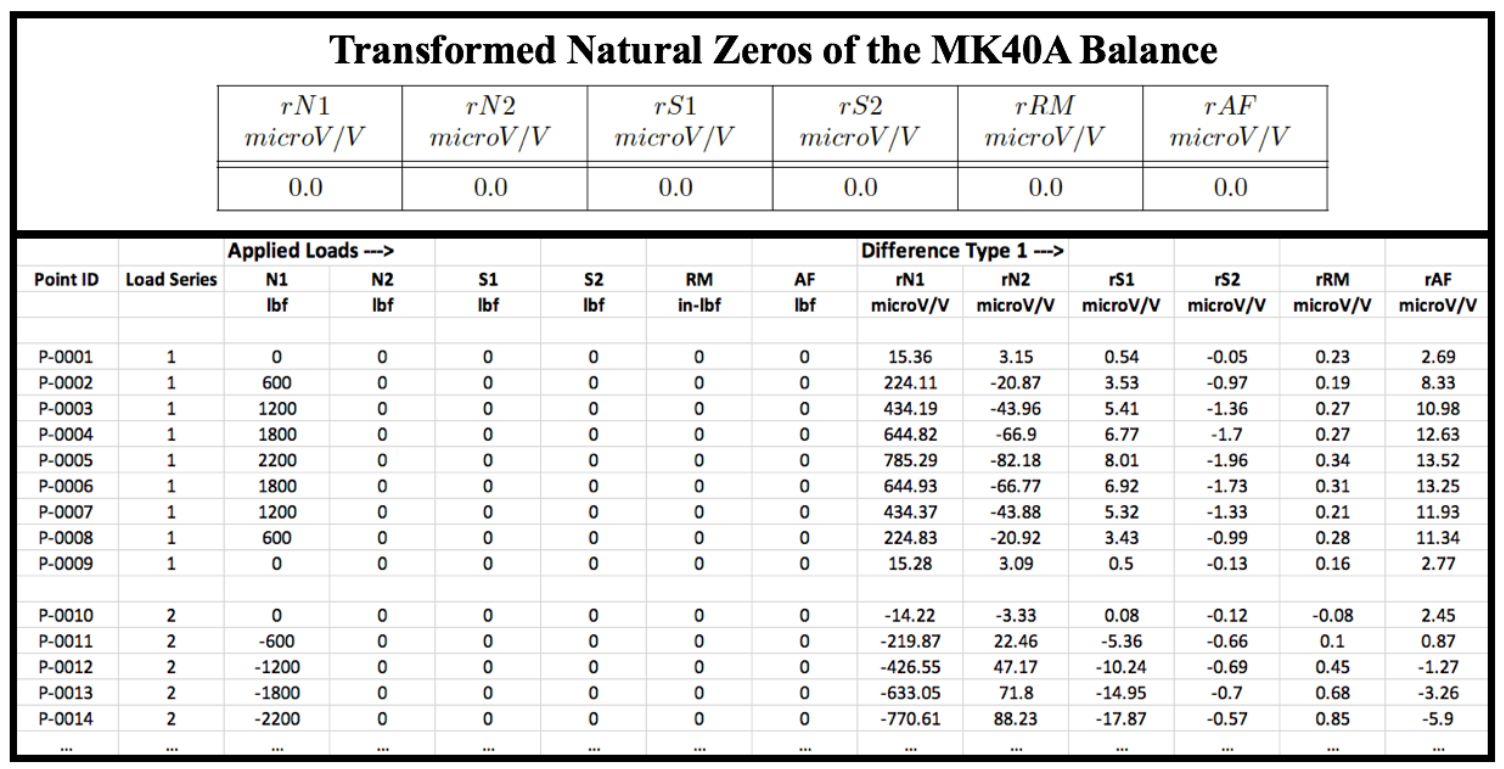

Fig. 3 Transformed natural zeros and revised calibration data of the MK40A balance if Difference Type 1 describes the "load state" of the balance in the "output space."

Figure 4 below shows the transformed natural zeros of the balance gages and parts of the revised calibration data input file of the balance when outputs were described in the format Difference Type 2.

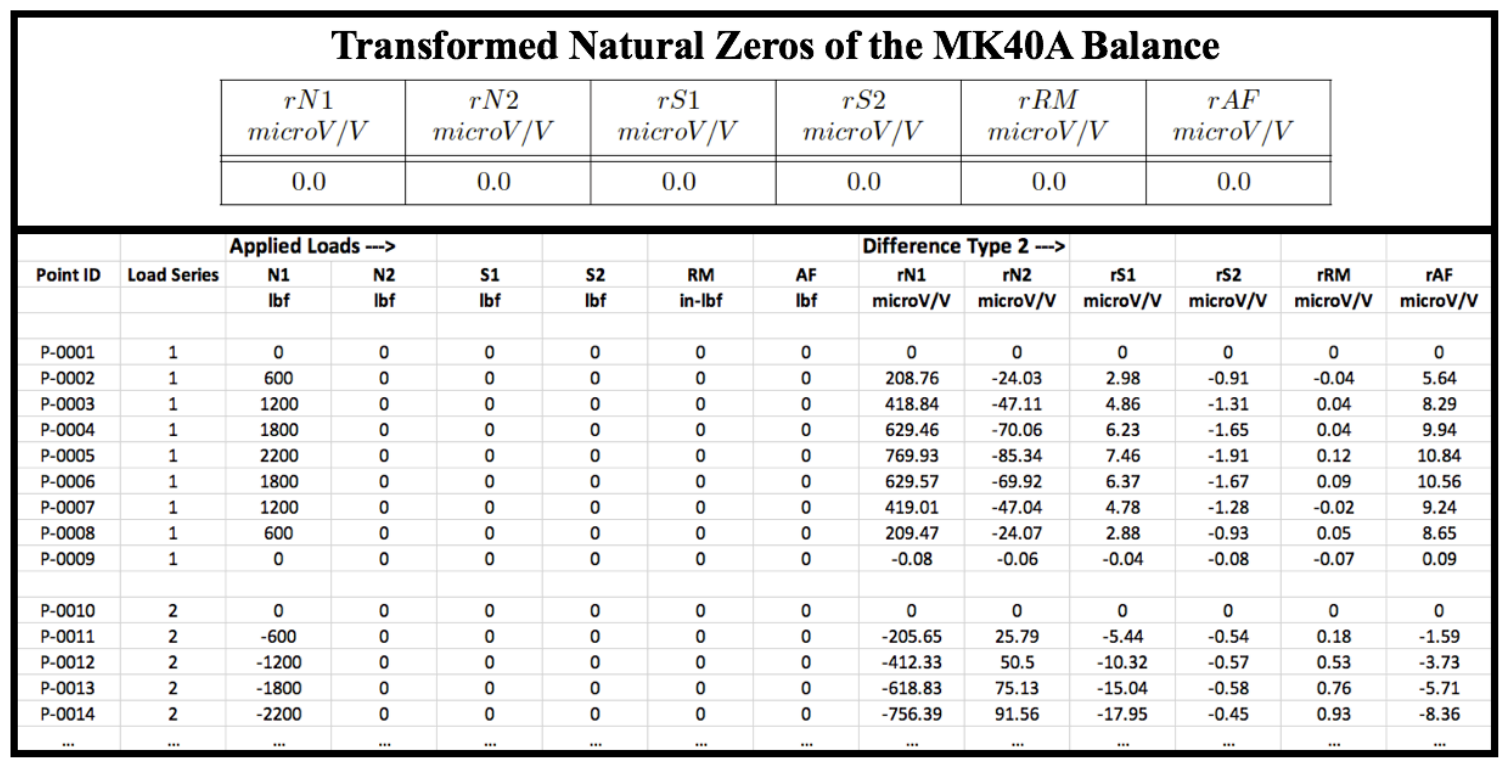

Fig. 4 Transformed natural zeros and revised calibration data of the MK40A balance if Difference Type 2 describes the "load state" of the balance in the "output space."

The last six data columns of Fig. 4 were obtained by subtracting the raw outputs of the zero load point of each load series (typically the outputs of the first point of each load series) from all other outputs of the corresponding load series. Therefore, for example, outputs of point P-0012 of Fig. 4 were obtained by subtracting the outputs of point P-0010 of Fig. 2, i.e, the outputs of the zero load point of load series 2, from the outputs of point $\mathrm{P}-0012$ of Fig. 2.

The final calibration data analysis was done in force balance format by separately applying the Iterative and the Non-Iterative Method to the calibration data. The entire analysis was performed by using NASA's BALFIT software package (see Ref. [8] for more detail). Absolute value terms were included in the regression model of the calibration data in all cases because the balance is known to have bi-directional normal and 
side force gage outputs. A total of 12 of the 16 load series were single-component loadings. Two sets of combined loadings were applied during the remaining 4 load series by placing gravity weights over the balance moment center. These loadings support the following cross-product terms: Iterative Method $\Longrightarrow(N 1 \cdot N 2)$, $(S 1 \cdot S 2) ;$ Non-Iterative Method $\Longrightarrow(r N 1 \cdot r N 2)$, and $(r S 1 \cdot r S 2)$. Therefore, the final regression models of both the outputs (Iterative Method) and the loads (Non-Iterative Method) had 21 terms for each component (intercept, six linear terms, six quadratic terms, two cross-product terms, and six absolute value terms). A tare load iteration was performed whenever feasible so that the weight of the metric part of the balance, of the calibration body, and of other calibration hardware pieces would be included in the final analysis.

\section{B. Iterative Method}

First, the Iterative Method was applied to the calibration data. Table 4 below shows the computed sensitivities of the five processing options (see Table 2) for this method.

Table 4: Sensitivities of the MK40A force balance in units of $[\mu V / V] /[l b f]$ or $[\mu V / V] /[i n-l b f]$.

\begin{tabular}{|c|c|c|c|c|c|c|}
\hline Option & $\frac{\partial r N 1}{\partial N 1}$ & $\frac{\partial r N 2}{\partial N 2}$ & $\frac{\partial r S 1}{\partial S 1}$ & $\frac{\partial r S 2}{\partial S 2}$ & $\frac{\partial r R M}{\partial R M}$ & $\frac{\partial r A F}{\partial A F}$ \\
\hline \hline 1 & 0.34599 & 0.37492 & 0.49057 & 0.50387 & 0.16361 & 3.56904 \\
\hline 2 & 0.34599 & 0.37492 & 0.49057 & 0.50387 & 0.16361 & 3.56904 \\
\hline 3 & 0.34599 & 0.37492 & 0.49057 & 0.50387 & 0.16361 & 3.56896 \\
\hline 4 & 0.34599 & 0.37493 & 0.49058 & 0.50389 & 0.16361 & 3.56881 \\
\hline 5 & 0.34599 & 0.37493 & 0.49058 & 0.50388 & 0.16361 & 3.56881 \\
\hline
\end{tabular}

Gage sensitivities show excellent agreement in all cases. It must be pointed out that it was only possible to include processing options 4 and 5 in the analysis because the maximum magnitude of the tare loads was less than the empirical threshold of $2 \%$ of the load capacity of the balance load components. Other calibration data sets cannot often be analyzed using options 4 and 5 because either the tare loads exceed the threshold of $2 \%$ or an analyst chooses to treat larger constant auxiliary loads as a part of the tare loads. The standard deviations of the calibration load residuals, i.e., of the difference between tare corrected and fitted loads, can also be compared. Table 5 below shows those values for all five processing options.

Table 5: Standard deviation of the load residuals of the MK40A balance in percent of capacity.

\begin{tabular}{|c|c|c|c|c|c|c|}
\hline Option & $N 1, \%$ & $N 2, \%$ & $S 1, \%$ & $S 2, \%$ & $R M, \%$ & $A F, \%$ \\
\hline \hline 1 & 0.0768 & 0.0712 & 0.0992 & 0.0904 & 0.0256 & 0.0696 \\
\hline 2 & 0.0768 & 0.0712 & 0.0992 & 0.0904 & 0.0256 & 0.0696 \\
\hline 3 & 0.0768 & 0.0712 & 0.0992 & 0.0904 & 0.0256 & 0.0696 \\
\hline 4 & 0.0768 & 0.0712 & 0.0994 & 0.0907 & 0.0256 & 0.0697 \\
\hline 5 & 0.0768 & 0.0712 & 0.0994 & 0.0907 & 0.0256 & 0.0698 \\
\hline
\end{tabular}

Again, the standard deviations of all options show excellent agreement.

\section{Non-Iterative Method}

In the next step, the Non-Iterative Method was applied to the calibration data set. Now, the calibration loads are directly fitted as a function of the outputs. A load iteration is not needed to predict loads from outputs during a wind tunnel test (see also Fig. 1b). Consequently, the regression coefficients of the primary gage outputs of the fitted loads are the inverse values of the sensitivities of the balance assuming that the sensitivity of a balance gage is defined as the partial derivative of a primary gage output with respect to the related primary load component. The computed inverse values of the sensitivities can be compared with corresponding values that were obtained after applying the Iterative Method to the data. Those values can be found on the principle diagonal of matrix $\mathbf{C}_{\mathbf{1}}^{-1}$ which defines the first part of the load iteration equation that the Iterative Method needs for the load prediction (for more detail see Ref. [2], pp. 18-19).

It was mentioned above that the MK40A balance has bi-directional gage outputs. Therefore, absolute value terms of the normal and side force gage outputs must be used in the regression models of the loads 
that the Non-Iterative Method uses. Consequently, Difference Type 1 was chosen for the analysis as the output format Raw Output cannot be chosen in this situation. This restriction on the use of the Raw Output format for the analysis of balances with bi-directional outputs is explained in detail in the paper's appendix. Processing option 2 of Table 2 was used for the analysis using the Non-Iterative Method and inverse values of the sensitivities of the six balance gages were compared with corresponding values for the Iterative Method. Table 6 below lists the inverse values of the sensitivities. The agreement between the inverse values of the

Table 6: Inverse values of the sensitivities of the MK40A in units of $[l b f] /[\mu V / V]$ or $[i n-l b f] /[\mu V / V]$.

\begin{tabular}{|c|c|c|c|c|c|c|}
\hline Method & $\frac{\partial N 1}{\partial r N 1}$ & $\frac{\partial N 2}{\partial r N 2}$ & $\frac{\partial S 1}{\partial r S 1}$ & $\frac{\partial S 2}{\partial r S 2}$ & $\frac{\partial R M}{\partial r R M}$ & $\frac{\partial A F}{\partial r A F}$ \\
\hline \hline Non-Iterative $^{2}$ & 2.89785 & 2.67451 & 2.04092 & 1.98755 & 6.11236 & 0.28020 \\
\hline Iterative $^{\dagger}$ & 2.89760 & 2.67413 & 2.03978 & 1.98585 & 6.11245 & 0.28021 \\
\hline
\end{tabular}

${ }^{\dagger}$ Inverse values of the sensitivities were directly taken from the principle diagonal of matrix $\mathbf{C}_{\mathbf{1}}^{-1}$.

sensitivities is excellent. Standard deviations of the calibration load residuals of the six load components of the balance were also computed and compared with values that were obtained by applying the Iterative Method. Table 7 below lists standard deviations for each load component and load prediction method:

Table 7: Standard deviation of the load residuals of the MK40A balance in percent of capacity

\begin{tabular}{|c|c|c|c|c|c|c|}
\hline Method & $N 1, \%$ & $N 2, \%$ & $S 1, \%$ & $S 2, \%$ & $R M, \%$ & $A F, \%$ \\
\hline \hline Non-Iterative & 0.0772 & 0.0715 & 0.0987 & 0.0904 & 0.0256 & 0.0691 \\
\hline Iterative & 0.0768 & 0.0712 & 0.0992 & 0.0904 & 0.0256 & 0.0696 \\
\hline
\end{tabular}

Again, the agreement between the standard deviations for the two methods is excellent. Overall, comparing results listed for the Non-Iterative Method and the Iterative Method in Table 6 and Table 7 above, it is concluded that the two methods have similar load prediction accuracies as long as (i) regression models with the same number and type of math terms are used for the analysis and (ii) the math terms have no hidden linear or massive near-linear dependency issues. It cannot be said that one method is better than the other because both methods use the same "information" about the physical behavior of the balance as input for the analysis. The "information" is hidden in the tare corrected loads and the measured electrical outputs of the calibration data of the balance. Small differences between the load predictions are expected because (i) the tare corrected loads are independently determined by each method, i.e., they are not exactly identical, and (ii) the methods use fundamentally different independent variable sets for the data analysis.

\section{Direct Measurement of the Natural Zeros}

In general, the natural zeros are defined as the raw electrical outputs of the balance gages in an assumed "weightless" condition (see Fig. 5a below). In other words, the natural zeros are the electrical representation

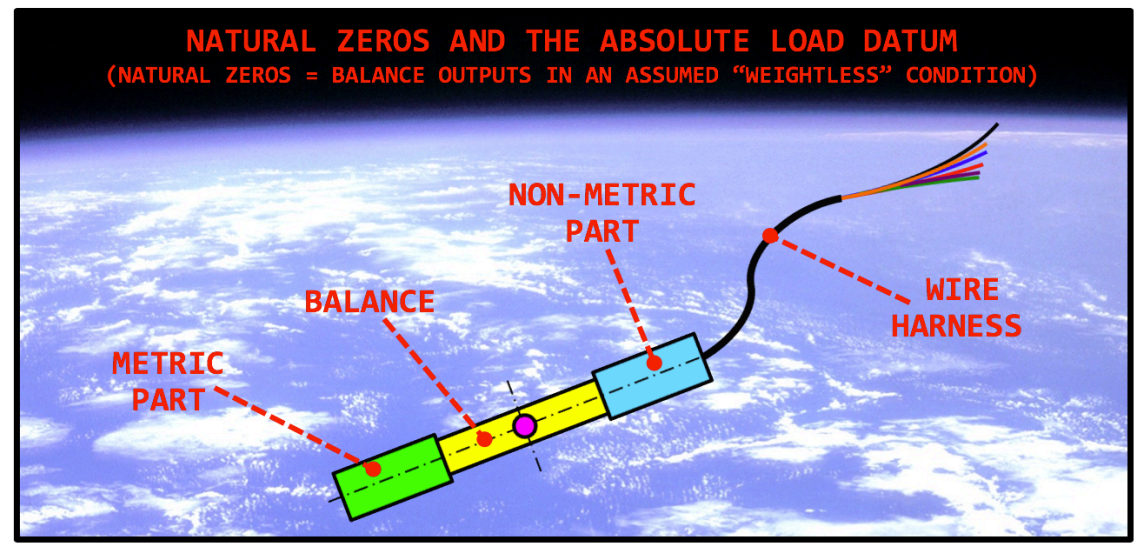

Fig. 5a Definition of the natural zeros as raw outputs in a "weightless" condition. 
of zero absolute load in the "output space" of the balance. Different methods are traditionally applied in aerospace testing to determine the natural zeros of balance gages. One approach, for example, attaches the balance to the calibration body, places the assembly four times on a leveling table such that each side of the calibration body is once in contact with the table's top surface, and takes the arithmetic mean of the corresponding four output measurements of each gage (see Fig. 5b below).

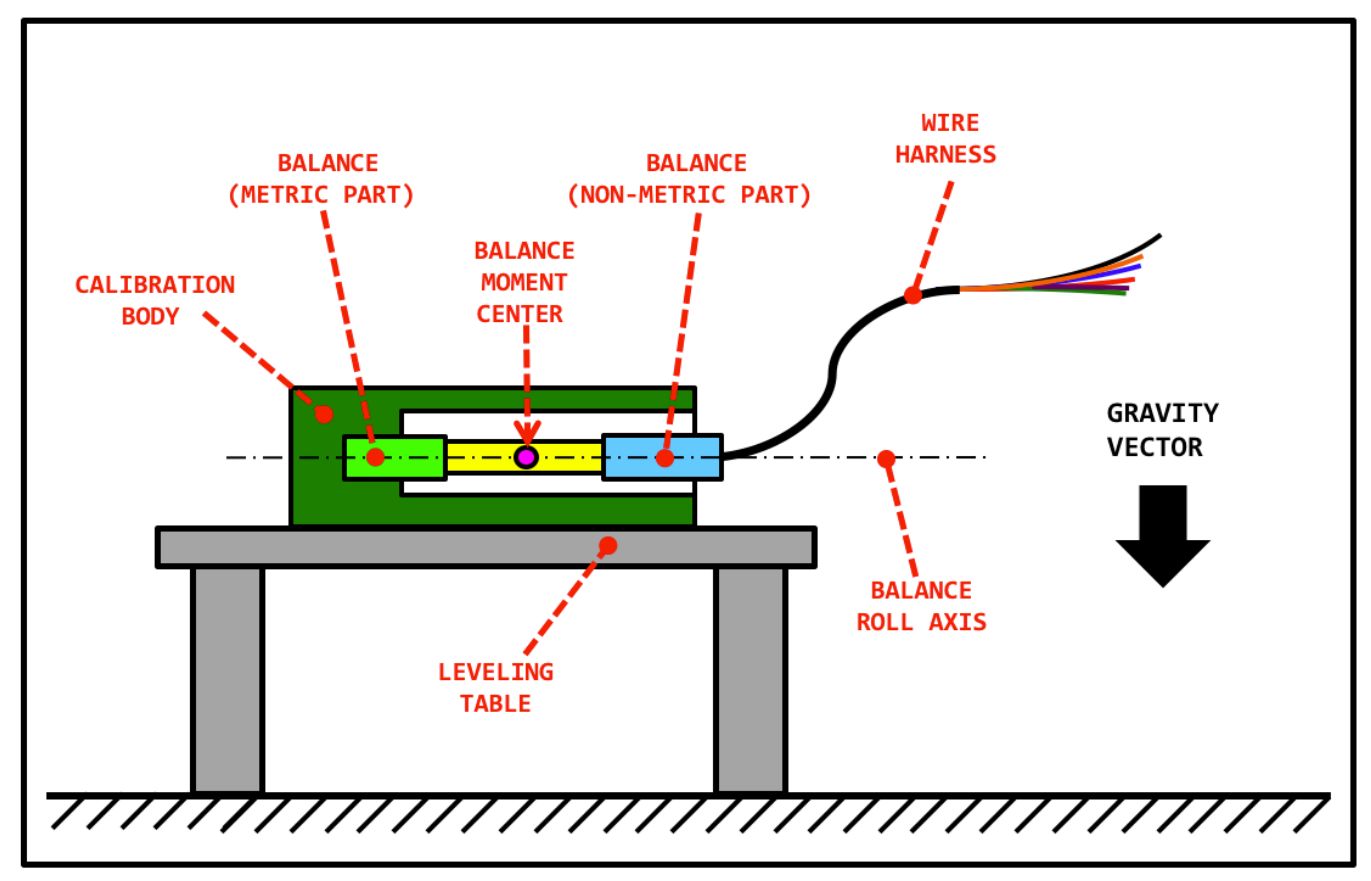

Fig. 5b Traditional setup for the experimental determination of the natural zeros of a balance.

It is also possible to use manual calibration data of the balance itself for a direct measurement of the natural zeros if (i) the Raw Outputs of the zero load points of each load series can be identified and (ii) the orientation of the balance and/or the calibration body relative to the gravitational acceleration is known for those data points. The direct measurement takes advantage of the fact that the raw electrical output of an "unloaded" gage must be very close or equal to its natural zero output. Table 8 below lists different cases for a typical force balance that may be used to directly measure the natural zeros of the balance gages (the direction of a balance load component relative to the gravitational acceleration describes the orientation of the balance; a "cross" marks a gage output that directly measures the natural zero).

Table 8: Force balance orientations that permit a direct measurement of the natural zeros.

\begin{tabular}{|c|c|c|c|c|c|c|}
\hline $\begin{array}{c}\text { Load component(s) acting in direction } \\
\text { of the gravitational acceleration }\end{array}$ & $r N 1$ & $r N 2$ & $r S 1$ & $r S 2$ & $r R M$ & $r A F$ \\
\hline \hline $\pm N 1$ and $\pm N 2$ & - & - & $\times$ & $\times$ & $\times$ & $\times$ \\
\hline $\pm S 1$ and $\pm S 2$ & $\times$ & $\times$ & - & - & $\times$ & $\times$ \\
\hline $\pm A F$ & $\times$ & $\times$ & $\times$ & $\times$ & $\times$ & - \\
\hline
\end{tabular}

The calibration data of the MK40A balance can be used to illustrate the direct measurement of the natural zeros whenever the outputs are given as Raw Outputs. Figure 5c below shows the typical setup in the NASA Ames Balance Calibration Laboratory that was used for the MK40A calibration before gravity weights were placed on the weight pans (the calibration data itself is partially shown in Fig. 2). 


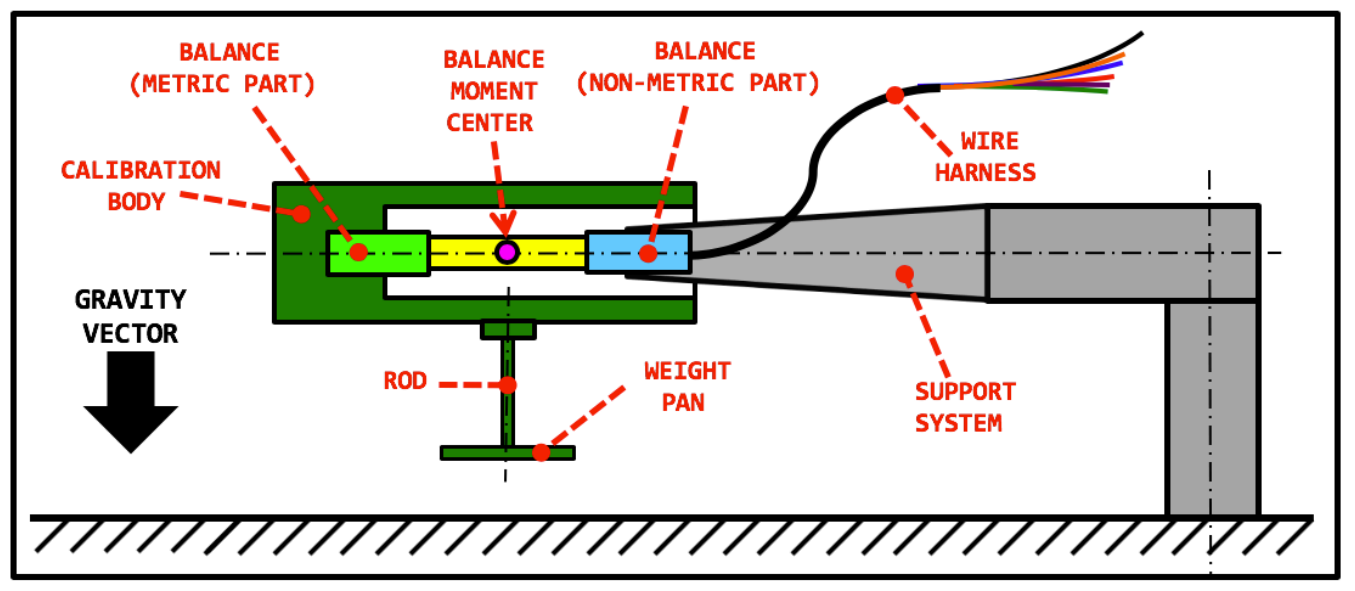

Fig. 5c Typical setup during manual calibration at the NASA Ames Balance Calibration Laboratory.

This setup shown in Fig. 5c above describes the balance load case that is responsible for the raw outputs of the zero load point of a load series. Figure 5d below shows the zero load points of three of the 16 load series of the calibration data that is shown in Fig. 2.

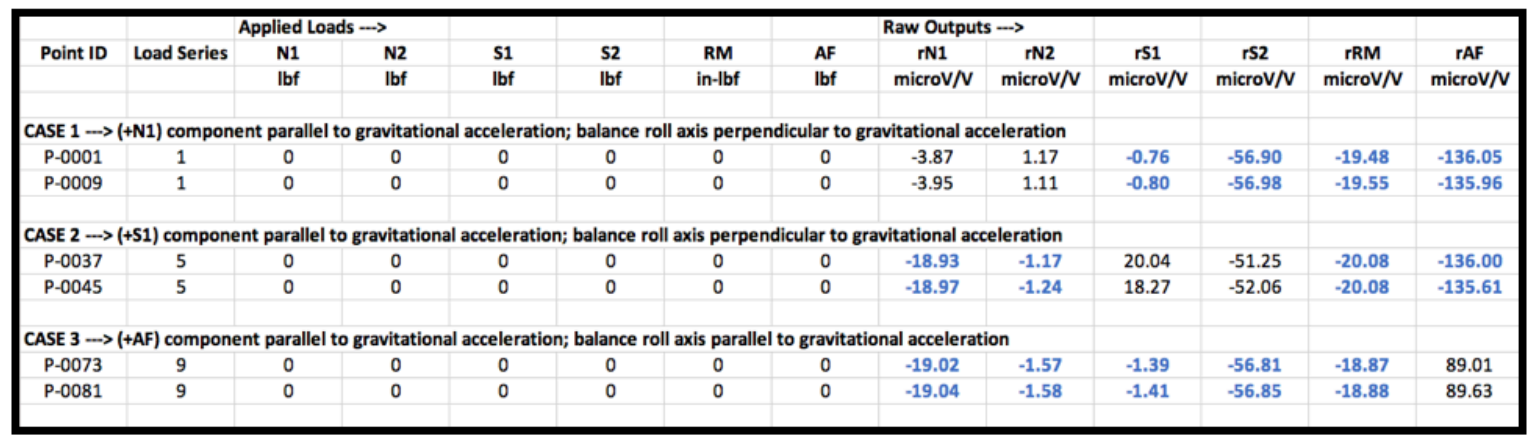

Fig. 5d The use of "unloaded" gages for the measurement of the natural zeros of the MK40A balance.

Now, outputs of "unloaded" gages need to be identified that are among the outputs of the zero load points of the calibration data. Load series 1, for example, "loaded" a positive forward normal force component. This situation is described by the first line in Table 8 above. Consequently, the two side force gages, the rolling moment gage, and the axial force gage of the balance must be "unloaded" gages. The electrical outputs of these four "unloaded" gages of load series 1 are highlighted in blue color in Fig. 5d. They must be the natural zeros of the corresponding gages if experimental error associated with balance alignment imperfections is temporarily ignored. Figure 5d also shows the "unloaded" outputs that were observed for two other load series.

It is interesting to compare the blue values shown in Fig. 5d with the natural zeros of the balance gages that are listed in Fig. 2. Those alternate values were obtained after averaging output sets of the balance that were measured after rotating the balance four times on a leveling table. In all cases, the difference between the natural zeros given in Fig. 2 and corresponding blue values in Fig. 5d is very small (within $\approx \pm 2 \mathrm{microV} / \mathrm{V})$.

\section{Summary}

Advantages and disadvantages of three different gage output formats for the analysis of wind tunnel strain-gage balance data were discussed. The first output format, i.e., Raw Output, works with balance data of all known output characteristics as long as (i) the Iterative Method is used for the load prediction and (ii) an intercept is included in the regression model of the outputs. In addition, the data input file 
preparation is both simple and easy to interpret as the outputs are not altered. It is also possible to use balance calibration data described in Raw Output format for a direct determination of the natural zeros of a balance. However, it must be pointed out that the Raw Output format cannot be chosen whenever calibration data of a balance with bi-directional outputs is analyzed by using the Non-Iterative Method.

The second output format, i.e., Difference Type 1, works with balance data of all known output characteristics if either the Iterative Method or the Non-Iterative Method is chosen for the load prediction. Predicted loads will exactly match those obtained with output format Raw Output as long as (i) the same set of math terms is used for the regression analysis of the outputs and (ii) no hidden linear or massive near-linear dependencies between math terms exist. The data input file preparation is simple as the outputs of the second output format option are obtained by subtracting the natural zeros of the balance gages from the raw outputs. It has to be pointed out that Difference Type 1 can be applied to balances with all known output characteristics independent of the chosen load prediction method. Therefore, Difference Type 1 can successfully be used for the analysis of data from a balance with bi-directional outputs if the Non-Iterative Method is chosen for the load prediction.

The third output format, i.e., Difference Type 2, also works with balance data of all known output characteristics if either the Iterative Method or the Non-Iterative Method is used for the load prediction. It is defined as the difference between the raw output of a gage and the output of the zero load point of the load series of the given data point. However, Difference Type 2 makes the implicit assumption that the tare loads are small and can be neglected. Therefore, load prediction results for Difference Type 2 are typically not as accurate as results for the two other output format choices. Gage outputs may have to be formatted using Difference Type 2 if a balance data analysis algorithm does not support one of the tare load iteration processes that are described in the literature (see, e.g., Ref. [2] or Ref. [5] for more details).

It must also be mentioned for completeness that a good understanding of the output format of a given balance data set is not just beneficial during the preparation of a data input file for an upcoming analysis task. It may also help an analyst to "decipher" third party balance data either to gain confidence in a supplied load prediction method or to perform an independent data analysis for troubleshooting purposes.

\section{Acknowledgements}

The author wants to thank Tom Volden of Jacobs Technology and Bob Gisler of NASA Ames Research Center for their critical and constructive review of the final manuscript. The work reported in this paper was supported by the Wind Tunnel Division at NASA Ames Research Center under contract NNA16BD26C.

\section{References}

[1] Ulbrich, N., "Comparison of Iterative and Non-Iterative Strain-Gage Balance Load Calculation Methods," AIAA 2010-4202, paper presented at the 27th AIAA Aerodynamic Measurement Technology and Ground Testing Conference, Chicago, Illinois, June/July 2010.

[2] AIAA/GTTC Internal Balance Technology Working Group, Recommended Practice - Calibration and Use of Internal Strain-Gage Balances with Application to Wind Tunnel Testing, AIAA R-091-2003, American Institute of Aeronautics and Astronautics, Reston, Virginia, 2003.

[3] Ulbrich, N., and Volden, T., "Strain-Gage Balance Calibration Analysis Using Automatically Selected Math Models," AIAA 2005-4084, paper presented at the 41st AIAA/ASME/SAE/ASEE Joint Propulsion Conference, Tucson, Arizona, July 2005.

[4] Ulbrich, N., and Volden, T., "Application of a New Calibration Analysis Process to the MK-III-C Balance," AIAA 2006-0517, paper presented at the 44th AIAA Aerospace Sciences Meeting, Reno, Nevada, January 2006.

[5] Ulbrich, N., "Regression Model Optimization for the Analysis of Experimental Data," AIAA 2009-1344, paper presented at the 47th AIAA Aerospace Sciences Meeting, Orlando, Florida, January 2009.

[6] Ulbrich, N., "A Universal Tare Load Prediction Algorithm for Strain-Gage Balance Calibration Data Analysis," AIAA 2011-6090, paper presented at the 47th AIAA/ASME/SAE/ASEE Joint Propulsion Conference, San Diego, California, July/August 2011.

American Institute of Aeronautics and Astronautics 
[7] Ulbrich, N., "Assessment of the Uniqueness of Strain-Gage Balance Load Predictions," AIAA 20164157, paper presented at the 32nd AIAA Aerodynamic Measurement Technology and Ground Testing Conference, Washington, D.C., June 2016.

[8] Kammeyer, M. E., and Ulbrich, N., "The Use of Absolute-Value Terms in Regression Modeling of MultiPiece Force Balances," AIAA 2015-3382, paper presented at the 31st AIAA Aerodynamic Measurement Technology and Ground Testing Conference, Dallas, Texas, June 2015.

[9] Ulbrich, N., and Volden, T., BALFIT - Software Tool for the Regression Analysis of Multivariate Data, User Guide (revised and corrected 4th edition), Jacobs Technology Inc., prepared for NASA Ames Research Center under contract NNA09DB39C, February 2016.

\section{Glossary}

bi-directional output $\equiv$ electrical output that changes slope near the origin if it is plotted versus the related load; absolute value terms are used in the regression model of balance data to model this characteristic.

gage sensitivity $\equiv$ partial derivative of a primary gage output with respect to the related primary gage load; for example, the forward normal force gage output of a force balance is a primary gage output and the forward normal force is the related primary gage load.

load series $\equiv$ subset of a balance calibration data set whose data points have the same tare loads.

natural zeros $\equiv$ balance gage outputs in an assumed weightless condition; they are the representation of zero absolute load in the output space.

primary gage load $\equiv$ load component that causes more than $\approx 80 \%$ of the output of the related balance gage; for example, the forward normal force is the primary gage load of the forward normal force gage.

primary gage output $\equiv$ gage output of a balance bridge that was placed on a balance flexure such that it would primarily respond to a single load component.

tare loads $\equiv$ balance loads that are caused by the weight of the metric part of the balance, the calibration body, and other calibration hardware pieces (yoke, rods, weight pans, etc.).

zero load point $\equiv$ data point of a load series whose outputs are exclusively caused by tare loads. 


\section{Appendix: Output Formats for Bi-directional Balance Data}

It is known in the aerospace testing community that the electrical outputs of balances of one specific design (ABLE/TASK) have the characteristic that the slope of four of its six primary gage outputs changes slightly when the sign of the related primary gage load changes (see Ref. [7] for more detail). Therefore, absolute value terms have to be included in the regression models of its calibration data in order to best model this repeatable behavior.

The bi-directional behavior of a balance gage can be illustrated by using data from NASA's MK40A force balance. In that case, the two normal force and the two side force gages are bi-directional. Figure 6a below shows, for example, the electrical output of the forward side force gage plotted versus the forward side force for the balance calibration data that was discussed earlier in the paper.

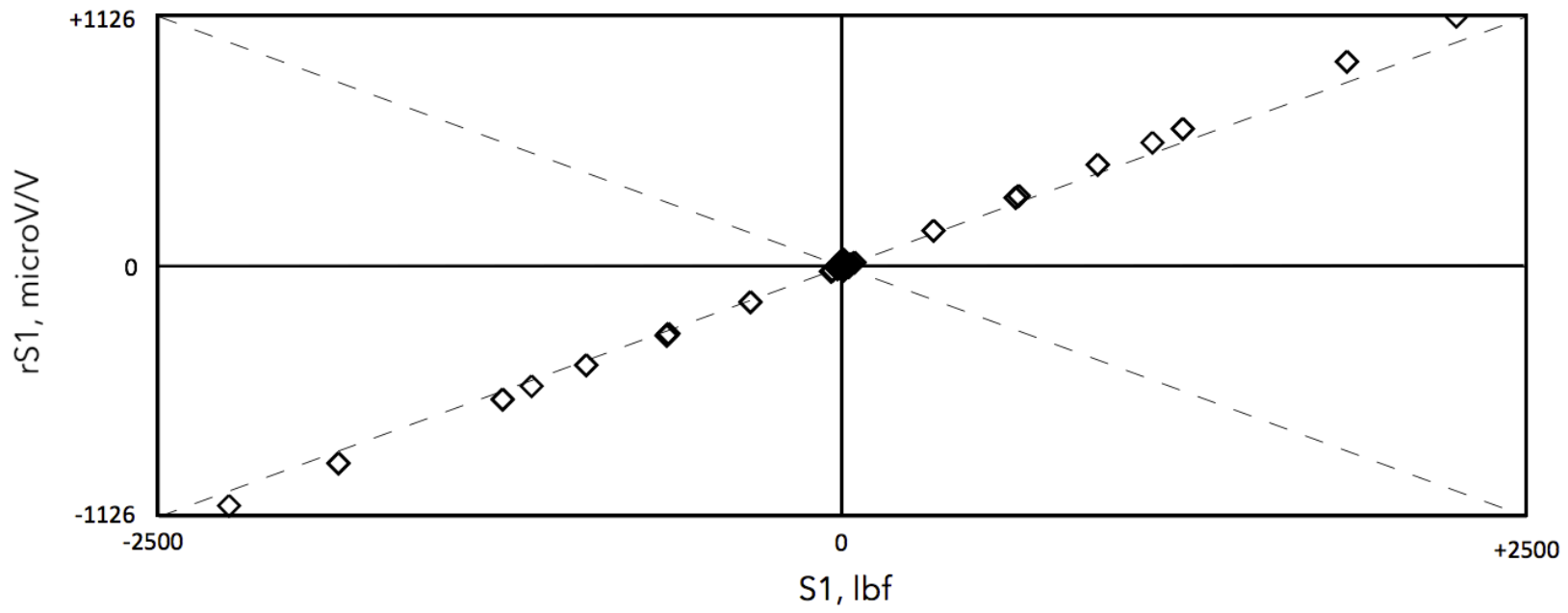

Fig. 6a The forward side force gage output plotted versus the forward side force of the MK40A balance.

It looks, superficially viewed, as if the data falls on a straight line. However, a closer examination reveals that the slope of the forward side force gage output changes slightly when the forward side force changes sign. This repeatable phenomenon amounts to about $22 \mathrm{microV} / \mathrm{V}$ at load capacity when the bi-directional characteristics of the forward side force gage are isolated and plotted versus the forward side force itself (for more detail see Fig. $6 \mathrm{~b}$ below).

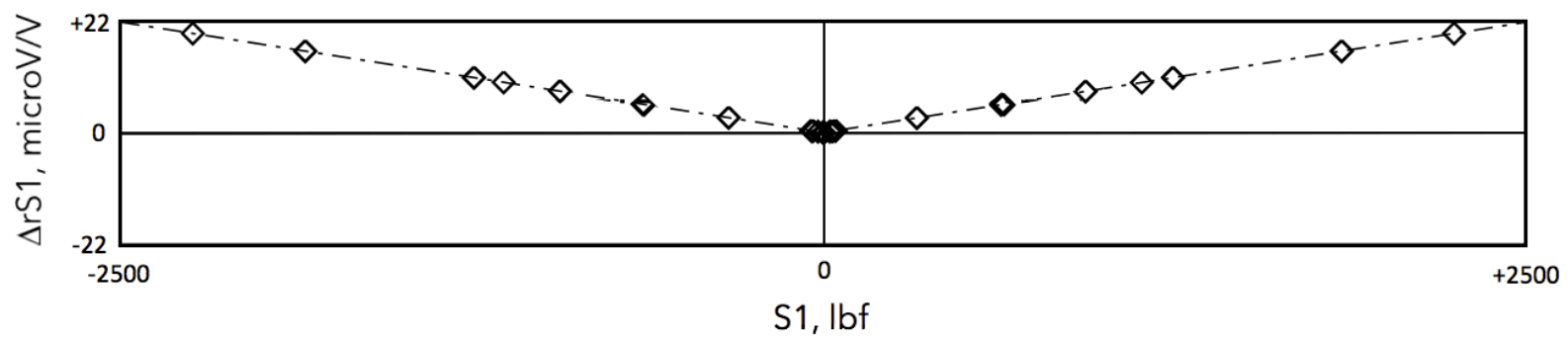

Fig. 6b Isolated bi-directional characteristics of the forward side force gage output.

The author's past experience showed that the gage output format can have a significant influence on the chosen load prediction method's ability to correctly model the behavior of a balance with bi-directional outputs. First, let us assume that an analyst chooses the Iterative Method for the load prediction. In that case, outputs are fitted as a function of loads by using the regression model that is defined in Eq. (3b). Furthermore, let us assume that the outputs are given as Raw Outputs. Then, the output of a bi-directional balance gage plotted versus the related primary gage load could look like the graph that is shown in Fig. 7a below. 


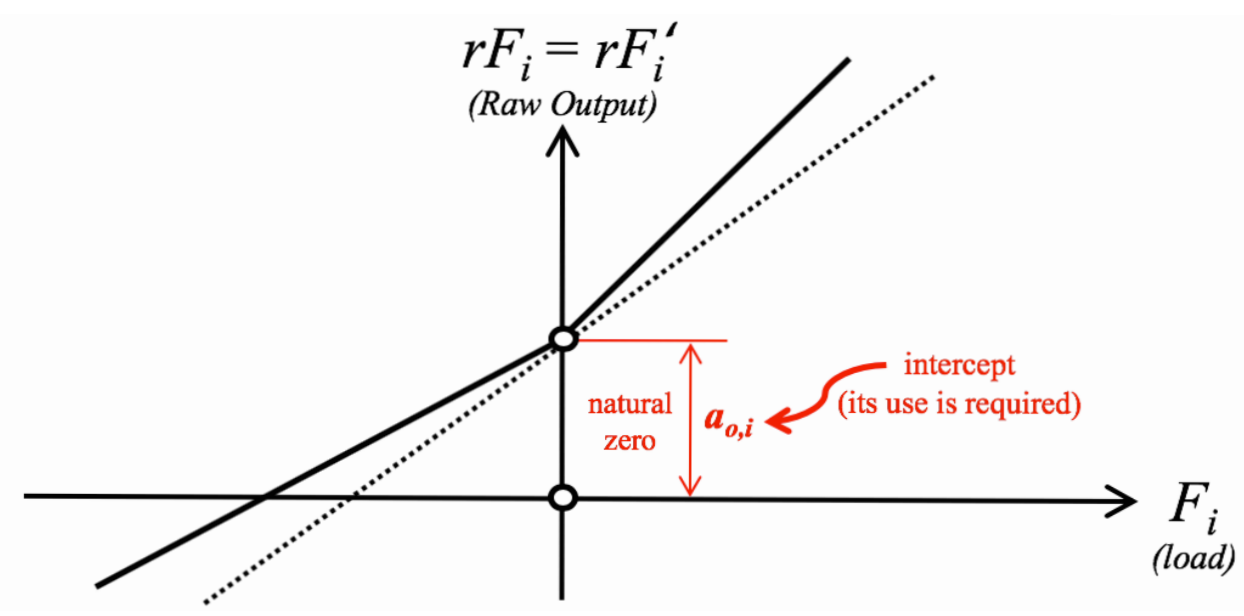

Fig. 7a Original output (Raw Output) plotted versus the primary load of a bi-directional gage.

The solid black line represents the functional relationship between electrical output and load. The vertical off-set of the solid line from the coordinate system origin corresponds to the natural zero of the given gage (this value is approximated by the intercept term in the regression model of the output). Now, it is assumed that (i) the outputs are shifted by the natural zero and that (ii) output format Difference Type 1 instead of Raw Output is used to analyze the balance data. Then, a plot of the transformed output of a bi-directional balance gage versus the related primary gage load could look like the graph that is shown in Fig. $7 \mathrm{~b}$ below.

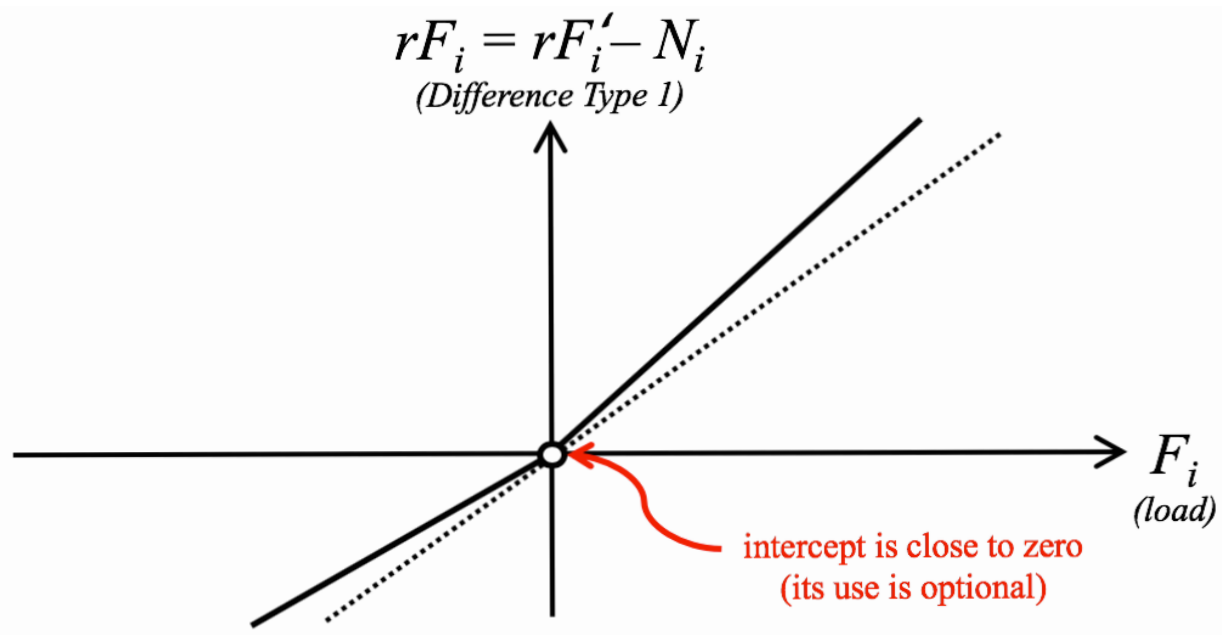

Fig. 7b Transformed output (Difference Type 1) plotted versus the primary load of a bi-directional gage.

The vertical off-set of the solid line from the origin has disappeared and the intercept term becomes an approximation of zero output. In principle, the two gage output characteristics shown in Figs. 7a and 7b can successfully be processed by using the Iterative Method whenever at least (i) the intercept term, (ii) the linear term $F_{i}$, and (iii) the absolute value term $\left|F_{i}\right|$ are contained in the regression model of the bi-directional output $r F_{i}$.

Now, let us assume that an analyst chooses the Non-Iterative instead of the Iterative Method for the load prediction. Then, the loads are fitted as a function of the outputs by using the regression model that is defined in Eq. (4b). The loads have become the dependent variable of the regression analysis and have to be plotted on the vertical axis of the plot that shows the characteristics of a bi-directional balance gage. A plot of the primary gage load of a bi-directional balance gage versus the related primary gage output could look like the graph that is shown in Fig. 8a below if the outputs are described using output format Raw Outputs. 


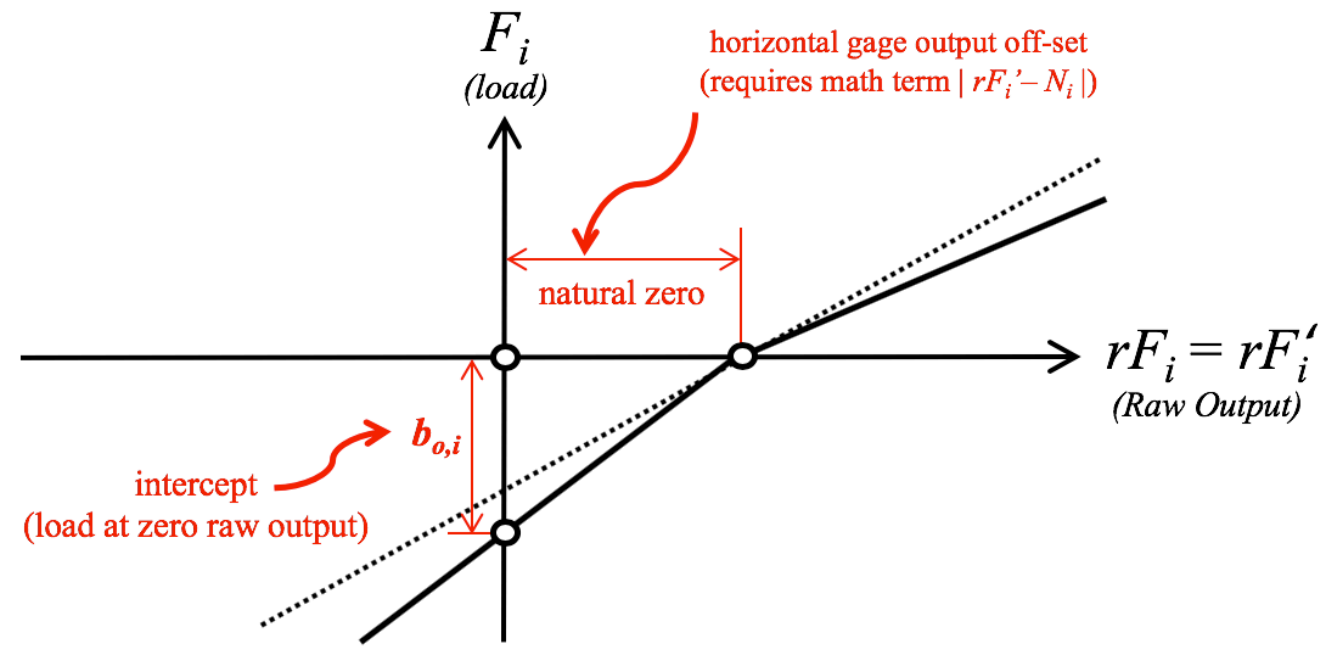

Fig. 8a Primary load of a bi-directional gage plotted versus the original output (Raw Output).

The horizontal off-set of the solid line from the origin corresponds to the natural zero of the gage. Consequently, a regression model term like $\left|r F_{i}-N_{i}\right|$ would be needed in the regression model of the load $F_{i}$ in order to model the bi-directional characteristics of the gage correctly. Unfortunately, this regression model term is not a part of the the traditional list of terms that were listed earlier. Therefore, the output format Raw Outputs is not recommended for use with the Non-Iterative Method whenever a balance has bi-directional outputs. The numerical errors associated with the combined use of the terms $r F_{i}$ and $\left|r F_{i}\right|$ instead of the terms $r F_{i}$ and $\left|r F_{i}-N_{i}\right|$ are expected to be too large.

Finally, it is assumed that output format Difference Type 1 instead of Raw Output is used to analyze the balance data. Then, a plot of the primary gage load versus the transformed output of a bi-directional balance gage could look like the graph that is shown in Fig. 8b below.

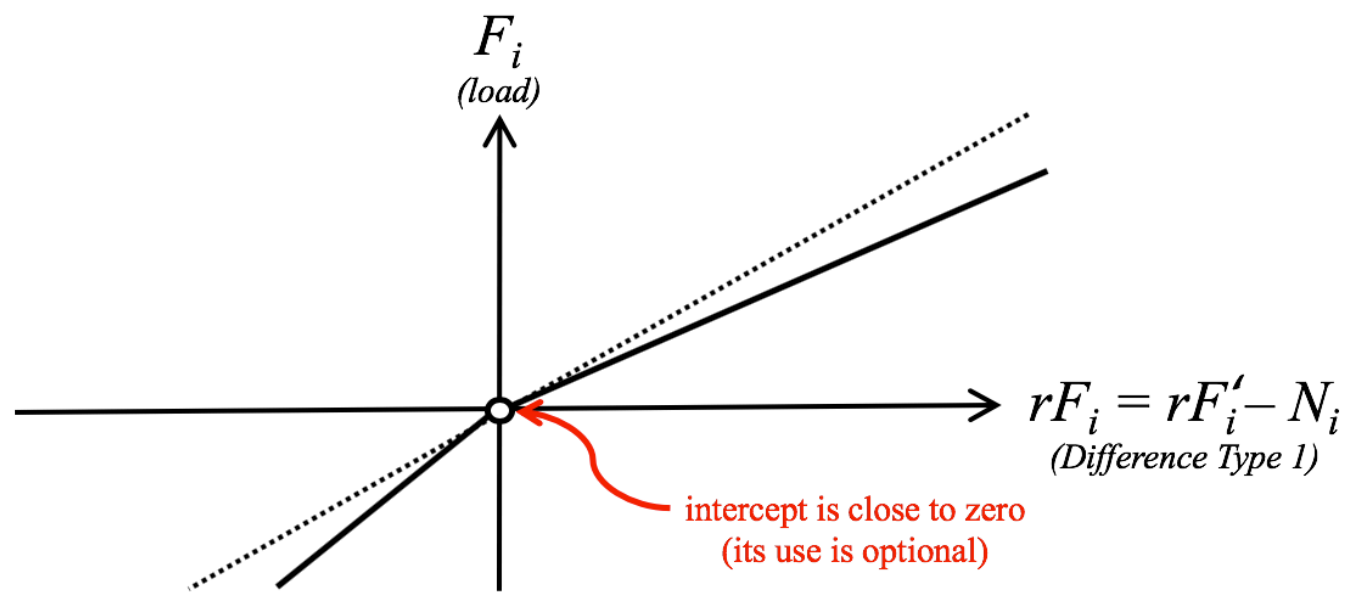

Fig. 8b Primary load of a bi-directional gage plotted versus the transformed output (Difference Type 1).

In that case, the horizontal off-set of the solid line from the origin has disappeared and the intercept term becomes an approximation of zero output. This situation can successfully be processed by using the Non-Iterative Method whenever (i) the linear term $r F_{i}$, and (ii) the absolute value term $\left|r F_{i}\right|$ are contained in the regression model of the primary gage load $F_{i}$. 